\title{
Approximate fixed point theorems of cyclical contraction mapping
}

\author{
S.A.M. MohSEnialhosseini
}

\begin{abstract}
Let $X_{i} ; i=1, \ldots, m$ are subsets of a metric space $X$ and also $T: \cup_{i=1}^{m} X_{i} \rightarrow \cup_{i=1}^{m} X_{i}$ and $T\left(X_{1}\right) \subseteq X_{2}, \ldots, T\left(X_{m-1}\right) \subseteq X_{m}, T\left(X_{m}\right) \subseteq$ $X_{1}$. We are going to consider element $x \in \cup_{i=1}^{m} X_{i}$ such that $d(x, T x) \leq \epsilon$ for some $\epsilon>0$. The existence results regarding approximate fixed points proved for the several operators such as Chatterjea and Zamfirescu on metric space (not necessarily complete). These results can be exploited to establish new approximate fixed point theorems for cyclical contraction maps. In addition, there is a new class of cyclical operators and contraction mapping on metric space (not necessarily complete) which do not need to be continuous. Finally, some examples are presented to illustrate our results for new approximate fixed point theorems on cyclical contraction maps.
\end{abstract}

\section{INTRODUCTION}

One of the most important results used in nonlinear analysis is the wellknown Banach's contraction principle which is a very popular tool in solving existing problems in many branches of Mathematical Analysis and its applications. Nowadays, there are plenty of problems in applied mathematics which can be solved by means of fixed point theory such as physics, chemistry, economics. In physics and engineering, fixed point technique has been used in areas like image retrieval, signal processing, and the existence and uniqueness of solutions for a class of nonlinear integral equations was studied. Still, practice proves that in many real situations an approximate solution is more than sufficient, so the existence of fixed points is not strictly required, but that of "nearly" fixed points.

In 1968, Kannan (see [1] [4] ) proved a fixed point theorem for operators which need not be continuous. A similar type of contractive condition has been studied by Chatterjea (see[3]). In 1972, by combining the above three independent contraction conditions, Zamfirescu (see [10]) obtained another fixed point result for operators which satisfy the following. In [1], the author

2000 Mathematics Subject Classification. Primary: 47H10; Secondary: 54H25.

Key words and phrases. Cyclical operators, Chatterjea operators, Diameter approximate fixed point. 
obtained a different contraction condition, also he formulated a corresponding fixed point theorem. In 2001, Rus (see [9]) defined $\alpha$-contraction, and in 2006, Berinde (see [2]) obtained some result on $\alpha$ - contraction for approximate fixed point on metric space.

In 2011, Mohsenalhosseini et al [6], introduced the approximate best proximity pairs and proved the approximate best proximity pairs property for it. Also, In 2012 , Mohsenalhosseini et al [7], introduced the approximate fixed point for completely norm space and map $T_{\alpha}$ and proved the approximate fixed point property for it. In 2014 , Mohsenalhosseini [8] introduced the Approximate best proximity pairs on metric space for contraction maps. This paper, on the other hand aims at introducing the new classes of cyclical operators and contraction maps (not necessarily continuous) regarding approximate fixed point on metric spaces.

\section{Preliminaries}

This section recalls the following notations and notions that will be used in what follows. In 2003, Kirk et al. [5], obtained an extension of Banach's fixed point theorem by considering a cyclical operator.

Definition 2.1 ([5]). Let $\left\{X_{i}\right\}_{i=1}^{m}$ be nonempty subsets of a complete metric space $X$. A mapping $T: \cup_{i=1}^{m} X_{i} \rightarrow \cup_{i=1}^{m} X_{i}$ satisfies the following condi$\operatorname{tion}\left(\right.$ where $\left.X_{i+1}=X_{1}\right)$

$$
T\left(X_{1}\right) \subseteq X_{2}, \ldots, T\left(X_{m-1}\right) \subseteq X_{m}, T\left(X_{m}\right) \subseteq X_{1},
$$

is called a cyclical operator.

Definition $2.2([7])$. Let $T: X \rightarrow X, \epsilon>0, x_{0} \in X$. Then $x_{0} \in X$ is an $\epsilon$-fixed point for $T$ if $\left\|T x_{0}-x_{0}\right\|<\epsilon$.

Remark 2.1 ([7]). In this paper the set of all $\epsilon$ - fixed points of $T$, for a given $\epsilon$, is denoted by:

$$
F_{\epsilon}(T)=\{x \in X \mid x \text { is an } \epsilon-\text { fixed point of } T\} .
$$

Definition 2.3 ([7]). Let $T: X \rightarrow X$. Then $T$ has the approximate fixed point property (a.f.p.p) if

$$
\forall \epsilon>0, F_{\epsilon}(T) \neq \varnothing .
$$

Theorem $2.1([7])$. Let $(X,\|\|$.$) be a completely norm space, T: X \rightarrow X$, $x_{0} \in X$ and $\epsilon>0$. If $\left\|T^{n}\left(x_{0}\right)-T^{n+k}\left(x_{0}\right)\right\| \rightarrow 0$ as $n \rightarrow \infty$ for some $k>0$, then $T^{k}$ has an $\epsilon-$ fixed point.

Now an important lemma by Berinde [2] regarding the existence of $\epsilon-$ fixed point of an operator is being recalled here.

Lemma 2.1 ([2]). Let $(X, d)$ be a metric space, $T: X \rightarrow X$ such that $T$ is asymptotically regular, i.e.,

$$
\lim _{n \rightarrow \infty} d\left(T^{n}(x), T^{n+1}(x)\right)=0, \quad \text { for all } x \in X .
$$


Then $T$ has the approximate fixed point property.

Lemma $2.2([2])$. Let $(X, d)$ be a metric space, $T: X \rightarrow X$ a operator and $\epsilon>0$. We assume that:

(i) $F_{\epsilon}(T) \neq \emptyset$;

(ii) $\forall \theta>0, \exists \phi(\theta)>0$ such that

$$
d(x, y)-d(T x, T y) \leq \theta \Rightarrow d(x, y) \leq \phi(\theta), \forall x, y \in F_{\epsilon}(T) \neq \emptyset .
$$

Then:

$$
\delta\left(F_{\epsilon}(T)\right) \leq \phi(2 \epsilon) .
$$

\section{Approximate FIXED POINT FOR CYCliCAL OPERATORS}

The section begins with three lemmas which will be used in order to prove all the results given in the second and in the third sections.

Definition 3.1. Let $\left\{X_{i}\right\}_{i=1}^{m}$ be nonempty susets of a metric space $X$. A cyclical operator $T: \cup_{i=1}^{m} X_{i} \rightarrow \cup_{i=1}^{m} X_{i}$ is said to be asymptotically regular at a point $x \in \cup_{i=1}^{m} X_{i}$, if

$$
\lim _{n \rightarrow \infty} d\left(T^{n}(x), T^{n+1}(x)\right)=0,
$$

where $T^{n}$ denotes the $n$th iterate of $T$ at $x$.

Lemma 3.1. Let $\left\{X_{i}\right\}_{i=1}^{m}$ be nonempty subsets of a metric space $X$ and $T: \cup_{i=1}^{m} X_{i} \rightarrow \cup_{i=1}^{m} X_{i}$ be a cyclical operator. Let $x_{0} \in \cup_{i=1}^{m} X_{i}$ and $\epsilon>0$. If $T: \cup_{i=1}^{m} X_{i} \rightarrow \cup_{i=1}^{m} X_{i}$ is asymptotically regular at each point $x_{0} \in \cup_{i=1}^{m} X_{i}$, then $T$ has an $\epsilon-$ fixed point.

Proof. Let $\epsilon>0$ be given and $x_{0} \in \cup_{i=1}^{m} X_{i}$ such that $\lim _{n \rightarrow \infty} d\left(T^{n} x_{0}, T^{n+1} x_{0}\right)=$ 0 , then there exists $N_{0}>0$ such that

$$
\forall n \geq N_{0}: d\left(T^{n} x_{0}, T^{n+1} x_{0}\right)<\epsilon .
$$

If $n=N_{0}$, then $d\left(T^{N_{0}}\left(x_{0}\right), T\left(T^{N_{0}}\left(x_{0}\right)\right)\right)<\epsilon$, and $T^{N_{0}}\left(x_{0}\right) \in F_{\epsilon}(T)$. So for each $\epsilon>0$ there exists an $\epsilon$-fixed point of $T$ in $\cup_{i=1}^{m} X_{i}$.

Lemma 3.2. Let $\left\{X_{i}\right\}_{i=1}^{m}$ be nonempty subsets of a metric space $X$ and $T: \cup_{i=1}^{m} X_{i} \rightarrow \cup_{i=1}^{m} X_{i}$ be a cyclical operator. Let $x_{0} \in \cup_{i=1}^{m} X_{i}$ and $\epsilon>0$. If $d\left(T^{n}\left(x_{0}\right), T^{n+k}\left(x_{0}\right)\right) \rightarrow 0$ as $n \rightarrow \infty$ for some $k>0$, then $T^{k}$ has an $\epsilon-$ fixed point.

Proof. The proof of Lemma is the same as the proof of Theorem 2.1 for $x \in \cup_{i=1}^{m} X_{i}$.

Definition 3.2. Let $\left\{X_{i}\right\}_{i=1}^{m}$ be nonempty closed subsets of a metric space $X, T: \cup_{i=1}^{m} X_{i} \rightarrow \cup_{i=1}^{m} X_{i}$ a cyclical operator and $\epsilon>0$. We define diameter of the set $F_{\epsilon}(T)$, i.e.,

$$
\delta\left(F_{\epsilon}(T)\right)=\sup \left\{d(x, y): x, y \in F_{\epsilon}(T)\right\} .
$$


Lemma 3.3. Let $\left\{X_{i}\right\}_{i=1}^{m}$ be nonempty subsets of a metric space $X, T$ : $\cup_{i=1}^{m} X_{i} \rightarrow \cup_{i=1}^{m} X_{i}$ a cyclical operator and $\epsilon>0$. We assume that:

(i) $F_{\epsilon}(T) \neq \emptyset$;

(ii) $\forall \theta>0, \exists \phi(\theta)>0$ such that

$$
d(x, y)-d(T x, T y) \leq \theta \Rightarrow d(x, y) \leq \phi(\theta), \quad \forall x, y \in F_{\epsilon}(T) \neq \emptyset .
$$

Then:

$$
\delta\left(F_{\epsilon}(T)\right) \leq \phi(2 \epsilon) .
$$

Proof. The proof of Lemma is the same as the proof of Lemma 2.2 for $x \in \cup_{i=1}^{m} X_{i}$.

\section{Approximate Fixed point FOR SEVERAL OPERATOR ON METRIC SPACES}

In this section a series of qualitative and quantitative results will be obtained regarding the properties of approximate fixed point. Also, by using Lemma 3.1 and 3.2 we prove approximate fixed point theorems for a new class of cyclical operators on metric spaces.

Definition 4.1. [9] A mapping $T: X \rightarrow X$ is a $\alpha$-contraction if there exists $\alpha \in(0,1)$ such that

$$
d(T x, T y) \leq \alpha d(x, y), \forall x, y \in X .
$$

Definition 4.2. Let $\left\{X_{i}\right\}_{i=1}^{m}$ be nonempty subsets of a metric space $X$, $T: \cup_{i=1}^{m} X_{i} \rightarrow \cup_{i=1}^{m} X_{i}$ is a $\alpha$-cyclical contraction if there exists $\alpha \in\left(0, \frac{1}{2}\right)$ such that

$$
d(T x, T y) \leq \alpha d(x, y) \quad \forall x \in X_{i}, y \in X_{i+1} .
$$

Theorem 4.1. Let $\left\{X_{i}\right\}_{i=1}^{m}$ be nonempty subsets of a metric space $X$ and Suppose $T: \cup_{i=1}^{m} X_{i} \rightarrow \cup_{i=1}^{m} X_{i}$ is a $\alpha$-cyclical contraction. Then $T$ has an $\epsilon$-fixed point.

Proof. Let $\epsilon>0$ and $x \in \cup_{i=1}^{m} X_{i}$.

$$
\begin{aligned}
d\left(T^{n} x, T^{n+1} x\right) & =d\left(T\left(T^{n-1} x\right), T\left(T^{n} x\right)\right) \\
& \leq \alpha d\left(T^{n-1} x, T^{n} x\right) \\
& \vdots \\
& \leq(\alpha)^{n} d(x, T x) .
\end{aligned}
$$

But $\alpha \in\left(0, \frac{1}{2}\right)$. Hence

$$
\lim _{n \rightarrow \infty} d\left(T^{n}, T^{n+1}\right)=0, \forall x \in \cup_{i=1}^{m} X_{i} .
$$

Hence by Lemma 3.1 it follows that $F_{\epsilon}(T) \neq \emptyset, \forall \epsilon>0$.

In 1972, Chatterjea (see [3]) considered another operator in which continuity is not imposed. Now, the approximate fixed point theorems by using cyclical operators are obtained. 
Definition 4.3. Let $\left\{X_{i}\right\}_{i=1}^{m}$ be nonempty subsets of a metric space $X, T$ : $\cup_{i=1}^{m} X_{i} \rightarrow \cup_{i=1}^{m} X_{i}$ is a Chatterjea cyclical operator if there exists $\alpha \in\left(0, \frac{1}{2}\right)$ such that

$$
d(T x, T y) \leq \alpha[d(x, T(y))+d(y, T(x))], \forall x, y \in \cup_{i=1}^{m} X_{i} .
$$

Theorem 2.4. Let $\left\{X_{i}\right\}_{i=1}^{m}$ be nonempty subsets of a metric space $X$. Suppose that the mapping $T: \cup_{i=1}^{m} X_{i} \rightarrow \cup_{i=1}^{m} X_{i}$ is a Chatterjea cyclical operator. Then $T$ has an $\epsilon$-fixed point.

Proof. Let $\epsilon>0$ and $x \in \cup_{i=1}^{m} X_{i}$.

$$
\begin{aligned}
d\left(T^{n} x, T^{n+1} x\right) & =d\left(T\left(T^{n-1} x\right), T\left(T^{n} x\right)\right) \\
& \leq \alpha\left[d\left(T^{n-1} x, T\left(T^{n} x\right)\right)+d\left(T^{n} x, T\left(T^{n-1} x\right)\right)\right] \\
& =\alpha\left[d\left(T^{n-1} x, T^{n+1} x\right)+d\left(T^{n} x, T^{n} x\right)\right]=\alpha d\left(T^{n-1} x, T^{n+1} x\right) .
\end{aligned}
$$

On the other hand

$$
d\left(T^{n-1} x, T^{n+1} x\right) \leq d\left(T^{n-1} x, T^{n} x\right)+d\left(T^{n} x, T^{n+1} x\right) .
$$

Then

$$
(1-\alpha) d\left(T^{n} x, T^{n+1} x\right) \leq \alpha d\left(T^{n-1} x, T^{n} x\right)
$$

hence

$$
\begin{aligned}
d\left(T^{n} x, T^{n+1} x\right) & \leq \frac{\alpha}{1-\alpha} d\left(T^{n-1} x, T^{n} x\right) \\
& \vdots \\
& \leq\left(\frac{\alpha}{1-\alpha}\right)^{n} d(x, T x) .
\end{aligned}
$$

But $\alpha \in\left(0, \frac{1}{2}\right)$ hence $\frac{\alpha}{1-\alpha} \in(0,1)$. Therefore

$$
\lim _{n \rightarrow \infty} d\left(T^{n} x, T^{n+1} x\right)=0, \quad \forall x \in \cup_{i=1}^{m} X_{i} .
$$

Now by Lemma 3.1 it follows that $F_{\epsilon}(T) \neq \emptyset, \forall \epsilon>0$.

Definition 4.4. Let $\left\{X_{i}\right\}_{i=1}^{m}$ be nonempty subsets of a metric space $X$, $T: \cup_{i=1}^{m} X_{i} \rightarrow \cup_{i=1}^{m} X_{i}$ is a Mohseni cyclical operator if there exists $\alpha \in\left(0, \frac{1}{2}\right)$ such that

$$
d(T x, T y) \leq \alpha[d(x, y)+d(T x, T y)] \quad \forall x \in X_{i}, y \in X_{i+1} .
$$

Theorem 4.2. Let $\left\{X_{i}\right\}_{i=1}^{m}$ be nonempty subsets of a metric space $X$ and Suppose $T: \cup_{i=1}^{m} X_{i} \rightarrow \cup_{i=1}^{m} X_{i}$ is a Mohseni cyclical operator. Then $T$ has an $\epsilon$-fixed point.

Proof. Let $\epsilon>0$ and $x \in \cup_{i=1}^{m} X_{i}$.

$$
\begin{aligned}
d\left(T^{n} x, T^{n+1} x\right) & =d\left(T\left(T^{n-1} x\right), T\left(T^{n} x\right)\right) \\
& \leq \alpha\left[d\left(T^{n-1} x, T^{n} x\right)+d\left(T^{n}, T^{n+1}\right)\right] .
\end{aligned}
$$


Therefore,

$$
(1-\alpha) d\left(T^{n} x, T^{n+1} x\right) \leq \alpha d\left(T^{n-1}, T^{n}\right) .
$$

So,

$$
\begin{aligned}
d\left(T^{n} x, T^{n+1} x\right) & \leq \frac{\alpha}{1-\alpha} d\left(T^{n-1}, T^{n}\right) \\
& \vdots \\
& \leq\left(\frac{\alpha}{1-\alpha}\right)^{n} d(x, T x) .
\end{aligned}
$$

But $\alpha \in\left(0, \frac{1}{2}\right)$, therefore $\left(\frac{\alpha}{1-\alpha}\right) \in(0,1)$. Hence

$$
\lim _{n \rightarrow \infty} d\left(T^{n}, T^{n+1}\right)=0, \quad \forall x \in \cup_{i=1}^{m} X_{i} .
$$

Hence by Lemma 3.1 it follows that $F_{\epsilon}(T) \neq \emptyset, \forall \epsilon>0$.

Example 4.1. Consider the sets: $X_{1}=\left\{\frac{1}{k}\right\}_{k=1}^{\infty} \cup\left\{\frac{-1}{2 k}\right\}_{k=1}^{\infty}$ and $X_{2}=\left\{\frac{-1}{k}\right\}_{k=1}^{\infty} \cup\left\{\frac{1}{2 k-1}\right\}_{k=1}^{\infty}$. Define the map $T: X_{1} \cup X_{2} \rightarrow X_{1} \cup X_{2}$ as

$$
T x= \begin{cases}\frac{-x}{x+4}, & \text { if } x \in X_{1} \\ \frac{-x}{4}, & \text { if } x \in X_{2} .\end{cases}
$$

It is easily to be checked that $T\left(X_{1}\right) \subseteq X_{2}$ and $T\left(X_{2}\right) \subseteq X_{1}$. For any $x \in X_{1}$ and $y \in X_{2}$ we have the chain of inequalities

$$
\begin{aligned}
d(T x, T y) & =\left|\frac{x}{x+4}-\frac{y}{4}\right| \leq \frac{1}{3}(|x|+|y|) \\
& \leq \frac{1}{3}\left(|x-y|+\left|\frac{x}{x+4}-\frac{y}{4}\right|\right) \\
& =\frac{1}{3}(d(x, y)+d(T x, T y)) .
\end{aligned}
$$

So $T$ satisfies all the conditions of Theorem 4.1 and thus it has a approximate fixed point.

Example 4.2. Let $X$ be a subset in $R$ endowed with the usual metric. Suppose $\left.\left.X_{1}=\right] 0,0.8\right]$ and $\left.\left.X_{2}=\right] 0, \frac{1}{2}\right]$. Define the map $T: X_{1} \cup X_{2} \rightarrow X_{1} \cup X_{2}$ as $T x=\frac{x}{4}$ for all $x \in X_{1} \cup X_{2}$ It is easily to be checked that $T\left(X_{1}\right) \subseteq X_{2}$ and $T\left(X_{2}\right) \subseteq X_{1}$. For any $x, y \in X_{1} \cup X_{2}$ we have the chain of inequalities

$$
\begin{aligned}
d(T x, T y)=\left|\frac{x}{4}-\frac{y}{4}\right| & \leq \frac{1}{3}\left(|x-y|+\left|\frac{x}{4}-\frac{y}{4}\right|\right) \\
& =\frac{1}{3}(d(x, y)+d(T x, T y)) .
\end{aligned}
$$


So $T$ satisfies all the conditions of Theorem 4.1 and thus for every $\epsilon>0$, $\left.F_{\epsilon}(T)\right) \neq \emptyset$. On the other hand take $0<\epsilon<\frac{1}{2}$ and select $x_{0} \in X_{1} \cup X_{2}$ such that $x_{0}<\frac{4}{3} \epsilon$. Then

$$
d(T x, x)=\left|\frac{x}{4}-x\right| \leq \epsilon .
$$

So $T$ has an approximate fixed point which implies that $\left.F_{\epsilon}(T)\right) \neq \emptyset$. On the contrary, there is no fixed point of $T$ in $X_{1} \cup X_{2}$.

By combining the three independent contraction conditions: $\alpha$-cyclical contraction, Mohseni cyclical, and Chatterjea cyclical operators we obtain another approximate fixed point result for operators which satisfy the following.

Definition 4.5. Let $\left\{X_{i}\right\}_{i=1}^{m}$ be nonempty subsets of a metric space $X$, $T: \cup_{i=1}^{m} X_{i} \rightarrow \cup_{i=1}^{m} X_{i}$ is a Mohsenialhosseini cyclical operator if there exists $\alpha, \beta, \gamma \in R, \alpha \in\left[0,1\left[, \beta \in\left[0, \frac{1}{2}\left[, \gamma \in\left[0, \frac{1}{2}\right.\right.\right.\right.\right.$ [ such that for all $x, y \in$ $\cup_{i=1}^{m} X_{i}$ at least one of the following is true:

(i) $d(T x, T y) \leq \alpha d(x, y)$;

(ii) $d(T x, T y) \leq \beta[d(x, y)+d(T x, T y)]$;

(iii) $d(T x, T y) \leq \gamma[d(x, T(y))+d(y, T(x))]$.

Theorem 4.3. Let $\left\{X_{i}\right\}_{i=1}^{m}$ be nonempty subsets of a metric space $X$ and Suppose $T: \cup_{i=1}^{m} X_{i} \rightarrow \cup_{i=1}^{m} X_{i}$ is a Mohsenialhosseini cyclical operator. Then $T$ has an $\epsilon$-fixed point.

Proof. Let $x, y \in \cup_{i=1}^{m} X_{i}$. Supposing $i i$ ) holds, we have that:

$$
\begin{aligned}
d(T x, T y) & \leq \beta[d(x, y)+d(T x, T y)] \\
& \leq \beta[d(x, T x)+d(T x, y)+d(T x, T y)] \\
& \leq \beta[d(x, T x)+d(T x, x)+d(x, y)+d(T x, T y)] \\
& =2 \beta d(x, T x)+\beta d(x, y)+\beta d(T x, T y)
\end{aligned}
$$

Thus

$$
d(T x, T y) \leq \frac{2 \beta}{1-\beta} d(x, T x)+\frac{\beta}{1-\beta} d(x, y)
$$

Supposing iii) holds, we have that:

$$
\begin{aligned}
d(T x, T y) & \leq \gamma[d(x, T y)+d(y, T x)] \\
& \leq \gamma[d(x, y)+d(y, T y)]+\gamma[d(y, T y)+d(T y, T x)] \\
& =\gamma d(T x, T y)+2 \gamma d(y, T y)+\gamma d(x, y) .
\end{aligned}
$$

Thus

$$
d(T x, T y) \leq \frac{2 \gamma}{1-\gamma} d(y, T y)+\frac{\gamma}{1-\gamma} d(x, y)
$$


Similarly:

$$
\begin{aligned}
d(T x, T y) & \leq \gamma[d(x, T y)+d(y, T x)] \\
& \leq \gamma[d(x, T x)+d(T x, T y)]+\gamma[d(y, x)+d(x, T x)] \\
& =\gamma d(T x, T y)+2 \gamma d(x, T x)+\gamma d(x, y) .
\end{aligned}
$$

Then

$$
d(T x, T y) \leq \frac{2 \gamma}{1-\gamma} d(x, T x)+\frac{\gamma}{1-\gamma} d(x, y) .
$$

Therefore for $T$ satisfying at least one of the conditions (i), (ii), (iii) we have that

$$
d(T x, T y) \leq 2 \eta d(x, T x)+\eta d(x, y)
$$

and

$$
d(T x, T y) \leq 2 \eta d(y, T y)+\eta d(x, y),
$$

where $\eta:=\max \left\{\alpha, \frac{\beta}{1-\beta}, \frac{\gamma}{1-\gamma}\right\}$, hold. Using these conditions implied by (i) (iii) and taking $x \in \cup_{i=1}^{m} X_{i}$, we have:

$$
\begin{aligned}
d\left(T^{n} x, T^{n+1} x\right) & =d\left(T\left(T^{n-1} x\right), T\left(T^{n} x\right)\right) \\
& \stackrel{(3.4)}{\leq} 2 \eta d\left(T^{n-1} x, T\left(T^{n-1} x\right)\right)+\eta d\left(T^{n-1} x, T^{n} x\right) \\
& =3 \eta d\left(T^{n-1} x, T^{n} x\right) .
\end{aligned}
$$

Then

$$
d\left(T^{n} x, T^{n+1} x\right) \leq \cdots \leq(3 \eta)^{n} d(x, T x)
$$

Therefore

$$
\lim _{n \rightarrow \infty} d\left(T^{n} x, T^{n+1} x\right)=0, \quad \forall x \in \cup_{i=1}^{m} X_{i} .
$$

Now by Lemma 3.1 it follows that $F_{\epsilon}(T) \neq \emptyset, \forall \epsilon>0$.

Example 4.3. Let $X=[0, \infty)$ and let $d$ be usual metric on $X$. Suppose $X_{1}=[0.1,2]$ and $X_{2}=[0.1,1]$. Fix $\beta \in(0,1)$ and define $T: X_{1} \cup X_{2} \rightarrow$ $X_{1} \cup X_{2}$ as

$$
T x= \begin{cases}0, & x \in[0,1-\beta), \\ \frac{x}{4}, & x \in[1-\beta, 1), \\ \frac{1-\beta}{4}, & x \in[1,2] .\end{cases}
$$

It is easy to check that $T\left(X_{1}\right) \subseteq X_{2}$ and $T\left(X_{2}\right) \subseteq X_{1}$. For any $x, y \in X_{1} \cup X_{2}$ there exists $\alpha \in\left(0, \frac{1}{2}\right)$ such that holds at least one of the condition Theorem 4.3. Thus by Theorem 4.3 for every $\epsilon>0, F_{\epsilon}(T) \neq \emptyset$. 
Definition 4.6. Let $\left\{X_{i}\right\}_{i=1}^{m}$ be nonempty subsets of a metric space $X$, $T: \cup_{i=1}^{m} X_{i} \rightarrow \cup_{i=1}^{m} X_{i}$ is a Mohseni-semi cyclical operator if there exists $\alpha \in] 0, \frac{1}{2}[$ such that

$$
d(T x, T y) \leq \alpha[d(x, y)+d(x, T(x))], \quad \forall x, y \in \cup_{i=1}^{m} X_{i} .
$$

Theorem 4.4. Let $\left\{X_{i}\right\}_{i=1}^{m}$ be nonempty subsets of a metric space $X$ and Suppose $T: \cup_{i=1}^{m} X_{i} \rightarrow \cup_{i=1}^{m} X_{i}$ is a Mohseni-semi cyclical operator. Then:

$$
\forall \epsilon>0, F_{\epsilon}(T) \neq \emptyset \text {. }
$$

Proof. Let $x \in \cup_{i=1}^{m} X_{i}$.

$$
\begin{aligned}
d\left(T^{n} x, T^{n+1} x\right) & =d\left(T\left(T^{n-1} x\right), T\left(T^{n} x\right)\right) \\
& \leq \alpha d\left(T^{n-1} x, T^{n} x\right)+\alpha d\left(T^{n-1} x, T^{n} x\right) \\
& =2 \alpha d\left(T^{n-1} x, T^{n} x\right) \leq \cdots \leq(2 \alpha)^{n} d(x, T x) .
\end{aligned}
$$

But $\alpha \in] 0, \frac{1}{2}[$. Therefore

$$
\lim _{n \rightarrow \infty} d\left(T^{n} x, T^{n+1} x\right)=0, \forall x \in \cup_{i=1}^{m} X_{i} .
$$

Now by Lemma 3.1, it follows that $F_{\epsilon}(T) \neq \emptyset, \forall \epsilon>0$.

Example 4.4. Let $X$ be a subset in $R$ endowed with the usual metric. Suppose $X_{1}=[0.01,0.8]$ and $X_{2}=\left[0.01, \frac{1}{2}\right]$. Define the map $T: X_{1} \cup X_{2} \rightarrow$ $X_{1} \cup X_{2}$ as $T x=\frac{x}{4}$ for all $x \in X_{1} \cup X_{2}$. It is easily to be checked that $T\left(X_{1}\right) \subseteq X_{2}$ and $T\left(X_{2}\right) \subseteq X_{1}$. For any $x, y \in X_{1} \cup X_{2}$ we have the chain of inequalities

$$
\begin{aligned}
d(T x, T y)=\left|\frac{x}{4}-\frac{y}{4}\right| & \leq \frac{1}{3}\left(|x-y|+\left|x-\frac{x}{4}\right|\right) \\
& =\frac{1}{3}(d(x, y)+d(x, T x)) .
\end{aligned}
$$

So $T$ satisfies all the conditions of Theorem 4.4 and thus for every $\epsilon>0$, $\left.F_{\epsilon}(T)\right) \neq \emptyset$.

\section{Diameter APproximate FiXed POINT FOR SEVERAL OPERATOR ON METRIC SPACES}

In this section, using Lemma 3.3, quantitative results for new cyclical operators will be formulated and proved, and some results regarding diameter approximate fixed point of such operators on metric spaces were given.

Theorem 5.1. Let $\left\{X_{i}\right\}_{i=1}^{m}$ be nonempty subsets of a metric space $X$. Suppose that $T: \cup_{i=1}^{m} X_{i} \rightarrow \cup_{i=1}^{m} X_{i}$ is a Mohseni cyclical operator. Then for every $\epsilon>0$,

$$
\delta\left(F_{\epsilon}(T)\right) \leq \frac{2 \epsilon(1+\alpha)}{1-2 \alpha} .
$$


Proof. Let $\epsilon>0$. and $x \in \cup_{i=1}^{m} X_{i}$. Condition (i) in Lemma 3.3 is satisfied, as one can see in the proof of Theorem 4.1. Now we only verify that condition (ii) in Lemma 3.3, holds.

Let $\theta>0$ and $x, y \in F_{\epsilon}(T)$, and assume that $d(x, y)-d(T x, T y) \leq \theta$. Then:

Therefore

$$
d(x, y) \leq \alpha[d(x, y)+d(T x, T y)]+\theta .
$$

$$
d(x, y) \leq \alpha[d(x, y)+d(T x, x)+d(x, y)+d(y, T y)]+\theta .
$$

As $x, y \in F_{\epsilon}(T)$, we know that

$$
d(x, T x) \leq \epsilon, \quad d(y, T y) \leq \epsilon .
$$

Therefore,

$$
d(x, y) \leq \frac{2 \alpha \epsilon+\theta}{1-2 \alpha} .
$$

So for every $\theta>0$ there exists $\phi(\theta)=\frac{2 \alpha \epsilon+\theta}{1-2 \alpha}>0$ such that

$$
d(x, y)-d(T x, T y) \leq \theta \quad \Rightarrow \quad d(x, y) \leq \phi(\theta) .
$$

Now by Lemma 3.3, it follows that

$$
\delta\left(F_{\epsilon}(T)\right) \leq \phi(2 \epsilon), \quad \forall \epsilon>0,
$$

which means exactly that

$$
\delta\left(F_{\epsilon}(T)\right) \leq \frac{2 \epsilon(1+\alpha)}{1-2 \alpha} .
$$

Example 5.1. Let $X$ be a subset in $R$ endowed with the usual metric. Suppose $X_{1}=[0.01,0.8]$ and $X_{2}=\left[0.01, \frac{1}{2}\right]$. Define the map $T: X_{1} \cup X_{2} \rightarrow$ $X_{1} \cup X_{2}$ as $T x=\frac{x}{4}$ for all $x \in X_{1} \cup X_{2}$.

By example $4.2 T: \cup_{i=1}^{m} X_{i} \rightarrow \cup_{i=1}^{m} X_{i}$ is a Mohseni cyclical operator. So $T$ satisfies all the conditions of Theorem 5.1 and thus for every $\epsilon>0$,

$$
\delta\left(F_{\epsilon}(T)\right) \leq \frac{2 \epsilon(1+\alpha)}{1-2 \alpha} .
$$

Theorem 5.2. Let $\left\{X_{i}\right\}_{i=1}^{m}$ be nonempty subsets of a metric space $X$. Suppose that $T: \cup_{i=1}^{m} X_{i} \rightarrow \cup_{i=1}^{m} X_{i}$ is a Mohsenialhosseini cyclical operator. Then for every $\epsilon>0$,

$$
\delta\left(F_{\epsilon}(T)\right) \leq 2 \epsilon \frac{1+\eta}{1-\eta},
$$

where $\eta:=\max \left\{\alpha, \frac{\beta}{1-\beta}, \frac{\gamma}{1-\gamma}\right\}$, and $\alpha, \beta, \gamma$ as in Definition 4.5

Proof. In the proof of Theorem 4.3, we have already shown that if $T$ satisfies at least one of the conditions (i), (ii), (iii) from Definition 4.5, then

$$
d(T x, T y) \leq 2 \eta d(x, T x)+\eta d(x, y),
$$


and

hold.

$$
d(T x, T y) \leq 2 \eta d(y, T y)+\eta d(x, y)
$$

Let $\epsilon>0$. We will only verify that condition (ii) in Lemma 3.3 is satisfied, as (i) holds, see the Proof of Theorem 4.3.

Let $\theta>0$ and $x, y \in F_{\epsilon}(T)$, and assume that $d(x, y)-d(T x, T y) \leq \theta$. Then

$$
\begin{aligned}
d(x, y) & \leq d(T x, T y)+\theta \Rightarrow \\
d(x, y) & \leq 2 \eta d(x, T x)+\eta d(x, y)+\theta \quad \Rightarrow \\
(1-\eta) d(x, y) & \leq 2 \eta \epsilon+\theta \Rightarrow \\
d(x, y) & \leq \frac{2 \eta \epsilon+\theta}{1-\eta} .
\end{aligned}
$$

So for every $\theta>0$ there exists $\phi(\theta)=\frac{2 \eta \epsilon+\theta}{1-\eta}>0$ such that

$$
d(x, y)-d(T x, T y) \leq \theta \quad \Rightarrow \quad d(x, y) \leq \phi(\theta) .
$$

Now by Lemma 3.3, it follows that

$$
\delta\left(F_{\epsilon}(T)\right) \leq \phi(2 \epsilon), \quad \forall \epsilon>0,
$$

which means exactly that

$$
\delta\left(F_{\epsilon}(T)\right) \leq 2 \epsilon \frac{1+\eta}{1-\eta}, \quad \forall \epsilon>0 .
$$

Example 5.2. Let $X=[0, \infty)$ and let $d$ be usual metric on $X$. Suppose $X_{1}=[0.1,2]$ and $X_{2}=[0.1,1]$. Fix $\beta \in(0,1)$ and define $T: X_{1} \cup X_{2} \rightarrow$ $X_{1} \cup X_{2}$ as

$$
T x= \begin{cases}0, & x \in[0,1-\beta), \\ \frac{x}{4}, & x \in[1-\beta, 1), \\ \frac{1-\beta}{4}, & x \in[1,2] .\end{cases}
$$

By Example 4.3, $T: \cup_{i=1}^{m} X_{i} \rightarrow \cup_{i=1}^{m} X_{i}$ is a Mohsenialhosseini cyclical operator. So, $T$ satisfies all the conditions of Theorem 5.2 and thus for every $\epsilon>0$, it is easy to check that $T\left(X_{1}\right) \subseteq X_{2}$ and $T\left(X_{2}\right) \subseteq X_{1}$.

For any $x, y \in X_{1} \cup X_{2}$ there exists $\alpha \in\left(0, \frac{1}{2}\right)$ such that holds at least one of the condition Theorem 4.3. Thus, by Theorem 4.3 for every $\epsilon>0$, $F_{\epsilon}(T) \neq \emptyset$.

Theorem 5.3. Let $\left\{X_{i}\right\}_{i=1}^{m}$ be nonempty subsets of a metric space $X$. Suppose that $T: \cup_{i=1}^{m} X_{i} \rightarrow \cup_{i=1}^{m} X_{i}$ is a Mohseni-semi cyclical operator. Then for every $\epsilon>0$

$$
\delta\left(F_{\epsilon}(T)\right) \leq \epsilon \frac{2+\alpha}{1-\alpha} .
$$


Proof. Let $\epsilon>0$. We will only verify that condition 2) in Lemma 3.3 is satisfied. Let $\theta>0$ and $x, y \in F_{\epsilon}(T)$, and assume that $d(x, y)-d(T x, T y) \leq$ $\theta$. Then

$$
\begin{aligned}
d(x, y) & \leq d(T x, T y)+\theta \quad \Rightarrow \\
d(x, y) & \leq \alpha[d(x, y)+d(x, T(x))]+\theta \quad \Rightarrow \\
(1-\alpha) d(x, y) & \leq \alpha d(x, T(x))+\theta \Rightarrow \\
d(x, y) & \leq \frac{\alpha \epsilon+\theta}{1-\alpha} .
\end{aligned}
$$

So, for every $\theta>0$ there exists $\phi(\theta)=\frac{\alpha \epsilon+\theta}{1-\alpha}>0$ such that

$$
d(x, y)-d(T x, T y) \leq \theta \quad \Rightarrow \quad d(x, y) \leq \phi(\theta) .
$$

Now by Lemma 3.3, it follows that

$$
\delta\left(F_{\epsilon}(T)\right) \leq \phi(2 \epsilon), \quad \forall \epsilon>0,
$$

which means exactly that

$$
\delta\left(F_{\epsilon}(T)\right) \leq \epsilon \frac{2+\alpha}{1-\alpha}, \quad \forall \epsilon>0 .
$$

Remark 5.1. Examples 4.2 and 4.3 holds in Theorem 5.3.

\section{REFERENCES}

[1] V. Berinde, On the Approximation of Fixed Points of Weak Contractive Mappings, Carpathian J. Math., 19 (2003), No. 1, 7-22.

[2] M. Berinde, Approximate fixed point theorems. Studia univ. "Babeş-Bolyai", Mathmatica, Volume LI, Number 1, March 2006.

[3] S.K. Chatterjea, Fixed-point Theorems, C.R. Acad. Bulgare Sci., 25 (1972), 727-730.

[4] R. Kannan, Some Results on Fixed Points, Bull. Calcutta Math. Soc., 10 (1968), 71-76.

[5] W.A. Kirk, P.S. Srinivasan, P. Veeramani, Fixed points for mappings satisfying cyclical contractive conditions, Fixed Point Theory Appl. 4 1, (2003), 79-89.

[6] S.A.M. Mohsenalhosseini, H. Mazaheri, M.A. Dehghan, Approximate best proximity pairs in metric space, Abstract and Applied Analysis, Volume 2011, Article ID 596971, 9 pages.

[7] S.A.M. Mohsenalhosseini, H. Mazaheri, Fixed Point for Completely Norm Space and Map $T_{\alpha}$, Mathematica Moravica 16-2, (2012), 25-35.

[8] S.A.M. Mohsenalhosseini, Approximate best proximity pairs on metric space for Contraction Maps, Advances in Fixed Point Theory 4 (2014), 310-324. 
[9] I.A. Rus, Generalized Contractions and Applications, Cluj University Press, ClujNapoca, 2001.

[10] T. Zamfirescu, Fixed Point Theorems in metric spaces, Arch. Math. (Basel), 23 (1972), 1292-298.

S. A. M. Mohsenialhosseini

FaCUlty of Mathematics

YAZD UNIVERSITY, YAZD

VALI-E-AsR University, Rafsanjan

IRAN

E-mail address: mohsenhosseini@yazd.ac.ir

amah@vru.ac.ir

mohsenialhosseini@gmail.com 